\title{
Staudt's Untersuchungen über das Pascal'sche Sechseck und einige sich daran anschliessende Bemerkungen.
}

Von L. Klug in Klausenburg.

Im 62. Bande des Crelle'schen Journals, in dem Aufsatze "Über die Steiner'schen Gegenpunkte ete." von Staudt befinden sich (Seite 147-150) zwei Sätze, laut welchen man Pascal'sche Sechsecke aus sechs, sich zu dreien in ein Steiner'sches Gegenpunktepaar schneidenden Pascal'schen Geraden construieren kann. Die Sätze sagen ferner, dass diesen $\infty^{1}$ Pascal'schen Sechsecken umschriebenen Kegelschnitte einem Büschel mit zwei Paar conjugiert-imaginären Grundpunkten angehören, und dass man in jedem Kegelschnitte des Bïschels zwei solche Sechsecke einbeschreiben

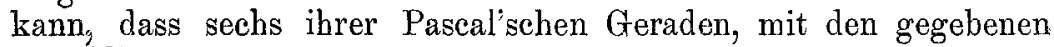
coincidieren.

Diese Sätze, welche auch noch andere Eigenschaften der Sechsecke, so wie der ihnen umschriebenen Kegelschnitte ausdrücken, sind an der citierten Stelle nicht bewiesen, oder wenigstens folgen sie nicht unmittelbar aus den Vorhergehenden. Ich habe desshalb versucht diese, bisher wenig beachteten Sätze, die im siebenten Punkte dieses Aufsatzes mit geänderter Bezeichnung wörtlich folgen, zu beweisen.

$$
* * *
$$

1. Auf einen Kegelschnitt $k$ nehmen wir sechs Punkte 123456 an. Dieselben sind die Eckpunkte von sechs einfachen Pascal'schen Sechsecken

$$
\begin{aligned}
& 163254=p^{\mathrm{I}} \quad 163452=\pi^{\mathrm{IV}} \\
& 123456=p^{\mathrm{rI}} \quad 123654=\pi^{\mathrm{V}} \\
& 143652=p^{\mathrm{III}} \quad 143256=\pi^{\mathrm{VI}}
\end{aligned}
$$

von deren Pascal'schen Geraden $p^{\mathrm{I}} p^{\mathrm{II}} p^{\mathrm{III}}$ und $\pi^{\mathrm{IV}} \pi^{\mathrm{V}} \pi^{\mathrm{VI}}$ sich in Steiner'sches Gegenpunktepaar 
schneiden.

$$
P=\left(p^{\mathrm{I}} p^{\mathrm{II}} p^{\mathrm{III}}\right), \quad \Pi=\left(\pi^{\mathrm{IV}} \pi^{\nabla} \pi^{\mathrm{VI}}\right)
$$

Durch $P$ und $\Pi$ II ziehen wir je drei Geraden $p^{\prime \text { I }} p^{\prime \text { II }} p^{\prime \text { III }}$, $\pi^{\mathrm{IV}} \pi^{\prime \mathrm{V}} \pi^{\prime \mathrm{VI}}$ derart, dass $p^{\mathrm{II}} p^{\mathrm{III}} p^{\mathrm{I}} p^{\mathrm{II}}, p^{\mathrm{III}} p^{\mathrm{I}} p^{\mathrm{II}} p^{\mathrm{II}}, p^{\mathrm{I}} p^{\mathrm{II}} p^{\mathrm{III}} p^{\prime \mathrm{III}}$ $\pi^{\mathrm{V}} \pi^{\mathrm{VI}} \pi^{\mathrm{IV}} \pi^{\prime \mathrm{IV}}, \pi^{\mathrm{VI}} \pi^{\mathrm{IV}} \pi^{\mathrm{V}} \pi^{\prime \mathrm{V}}, \pi^{\mathrm{IV}} \pi^{\mathrm{V}} \pi^{\mathrm{VI}} \pi^{\prime \mathrm{VI}}$ harmonische Würfe bilden sollen.

Wird zur Abkürzung der Punkte $\left(p^{\prime i} \pi^{\prime}\right)$ mit $\left(p^{i} \pi^{l}\right)^{\prime}$ bezeichnet, dann lässt sieh zeigen, dass

$$
\begin{aligned}
& \left(p^{\mathrm{I}} \pi^{\mathrm{IV}}\right)^{\prime} \text { auf der Geraden 56, }\left(p^{\mathrm{II}} \pi^{\mathrm{IV}}\right)^{\prime} \text { auf der Geraden 14, }
\end{aligned}
$$

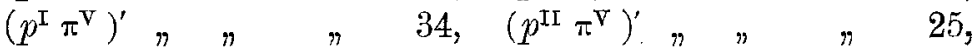

$$
\begin{aligned}
& \left(p^{\mathrm{I}} \pi^{\mathrm{VI}}\right)^{\prime} \quad " \quad n \quad n \quad 12,\left(p^{\mathrm{II}} \pi^{\mathrm{VI}}\right)^{\prime} \quad n \quad n \quad n \quad n \quad 36 \text {, } \\
& \left(p^{\mathrm{III}} \pi^{\mathrm{IV}}\right)^{\prime} \text { auf der Geraden 23, } \\
& \left(p^{\mathrm{III}} \pi^{\mathrm{V}}\right)^{\prime} \quad n \quad n \quad n \quad 16 \text {, } \\
& \left(p^{\mathrm{III}} \pi^{\mathrm{VI}}\right)^{\prime} \quad n \quad n \quad n \quad 45
\end{aligned}
$$

liegt.

Es sind nämlich

$p^{\mathrm{I}}, \pi^{\mathrm{IV}}, 56$ die Diagonalen des Viereckes $\left[\begin{array}{llll}16 & 25 & 45 & 36\end{array}\right]$, $p^{\mathrm{I}}, \pi^{\mathrm{V}}, 34 \quad " \quad " \quad\left[\begin{array}{llll}14 & 23 & 45 & 36\end{array}\right]$,

also wird die Diagonale $p^{\mathrm{I}}$ die Punkte $\left(\pi^{\mathrm{IV}}, 56\right),\left(\pi^{\mathrm{V}}, 36\right)$ so wie ihre Verbindungslinie harmonisch trennen von 45,36 . Aber die Schnittpunkte $(12,45),(12,36)$ liegen bezw. auf $p^{\mathrm{II}}, p^{\mathrm{III}}$, also geht die Gerade $p^{\prime \text { I }}$ durch den Schnittpunkt von 12 mit der Geraden $\left|\left(\pi^{\mathrm{IV}}, 56\right)\left(\pi^{\mathrm{V}}, 34\right)\right|$. Dieser Schnittpunkt ist aber ein Diagonalpunkt des Vierseits $\left[\pi^{\mathrm{TV}} \pi^{\mathrm{V}} 5634\right]$ welcher daher die Diagonale $\pi^{\mathrm{VI}}$ von den Seiten $\pi^{\mathrm{VI}} \pi^{\mathrm{V}}$ harmonisch trennt und als solcher auf der Geraden $\pi^{\prime \mathrm{VI}}$ liegt. Der Punkt $\left(p^{\mathrm{I}} \pi^{\mathrm{VI}}\right)^{\prime}$ ist daher schließlich ein Punkt der Geraden 12.

Nachdem der Punkt $\left(p^{\mathrm{I}} \pi^{\nabla \mathrm{I}}\right)^{\prime}$ die Gerade $p^{\mathrm{I}}$ von 45,36 und die Gerade $\pi^{\mathrm{VI}}$ von 34,56 harmonisch trennt, so trennen die Punkte $\left(p^{\mathrm{I}} \pi^{\mathrm{VI}}\right),\left(p^{\mathrm{I}} \pi^{\mathrm{VI}}\right)^{\prime}$ sämmtliche Gegenseiten des Viereckes 3456 harmonisch und sind daher conjugierte Pole in Hinsicht aut den Kegelschnitt $k$. Bezeichnet man also mit Staudt solche Punkte wie $\left(p^{i} \pi^{l}\right),\left(p^{i} \pi^{l}\right)^{\prime}$ gegenüberliegend, so hat man:

Die Pascal'schen Geraden $p^{\mathrm{I}} p^{\text {Ir }} p^{\text {IIr }}$ der Sechsecke $163254,123456,143652$ schneiden die Pascal'schen Geraden $\pi^{\mathrm{IV}} \pi^{\mathrm{V}} \pi^{\mathrm{VI}}$ der Sechsecke 163452,123654 , 143256 in neun Punkten, und die drei Geraden $\left(p^{\mathrm{I}} p^{\mathrm{II}} p^{\Pi \mathrm{IT}}\right)^{\prime}$, welche je zwei der drei ersten Pascal'schen Geraden von der dritten harmonisch trennen, schneidendie drei Geraden $\left(\pi^{\mathrm{IV}} \pi^{\mathrm{V}} \pi^{\mathrm{VI}}\right)^{\prime}$, welche je zwei der anderen Pascal'schen Geraden von der dritten harmonischtrennen, ebenfalls in neun Punkten. Von diesen achtzehn Schnittpunkten sind je zwei gegenüberliegende, wie $\left(p^{i} \pi^{l}\right),\left(p^{i} \pi^{l}\right)^{\prime}$, conjugierte Pole in 
Hinsicht auf den, dem Pascal'schen Sechseck 123456 umschriebenen Kegelschnitt $k$. Die neun letzteren Schnittpunkte $\left(p^{i} \pi^{l}\right)^{\prime}$ liegen auf denjenigen neun Geraden, welche die Eckpunkte des Dreieckes 135 mit den Eckpunkten des Dreieckes 246 verbinden.

2. Die Strahlenpaare $p^{\mathrm{I}} p^{\mathrm{II}}, p^{\mathrm{II}} p^{\prime \mathrm{II}}, p^{\mathrm{III}} p^{\text {III }}$ und $\pi^{\mathrm{IV}} \pi^{\mathrm{IV}}$, $\pi^{\mathrm{V}} \pi^{\prime \mathrm{V}}, \pi^{\mathrm{VI}} \pi^{\prime \mathrm{VI}}$ sind conjugierte Strahlen einer Involution $J_{p}$ und einer Involution $J_{\pi}$. Es seien $p^{t} p^{t^{\prime}}$ und $\pi^{\prime \prime} \pi^{\prime u}$ zwei beliebige Paare conjugierte Strahlen der involutorischen Büschel $J_{p}, J_{\pi}$, und $l$ möge irgend einen der Zeiger IV, V, VI bedeuten.

Wegen $J_{p}$ ist

$$
\pi^{i}\left(p^{\mathrm{I}} p^{\mathrm{II}} p^{\mathrm{III}} p^{t}\right) \bar{\wedge} \pi^{t^{l}}\left(p^{\mathrm{I}} p^{\mathrm{II}} p^{\mathrm{III}} p^{t}\right)^{\prime}
$$

und da die drei ersten Paare entsprechender Punkte conjugierte Pole sind in Hinsicht auf $k$, so sind es auch die Punkte $\left(\pi^{l} p^{t}\right)$, $\left(\pi^{l} p^{t}\right)^{\prime}$. Daher werden in der projectiven Beziehung

$$
p^{i}\left(\pi^{\mathrm{VV}} \pi^{\mathrm{V}} \pi^{\mathrm{Vr}} \pi^{i b}\right) \pi p^{\prime t}\left(\pi^{\mathrm{IV}} \pi^{\mathrm{V}} \pi^{\mathrm{VI}} \pi^{u}\right)^{i}
$$

die drei ersten, also auch das letzte entsprechende Punktepaar conjugierten Pole sein in Hinsicht auf $k$. D. h.:

Zwei beliebige conjugierte Strahlen des involutorischen Büschels $J_{p}$, schneiden $z$ wei beliebige conjugierte Strahlen des involutorischen Büschels $J_{\pi}$, in conjugierten Polen in Hinsicht auf $k$.

Davon haben wir als besonderen Fall:

Die conjugiert-imaginären Doppelstrahlen $x x_{1}$, $y y_{1}$ der involutorischen Büschel $J_{p}, J_{\pi}$ sehneiden sich auf den Kegelschnittl.

Oder allgemeiner gefasst:

Diejenigen conjugiert-imaginären Strahlenpare, welche mit den durch ein Steiner'sches Gogenpunktepaargehenden Pascal'schen Geraden äquianharmonisch liegen, schneiden sich auf den, dem Pascal'schen Sechseck umschriebenen Kegelschnitt.

Anmerkung. Trennen in $J_{p}$ die conjugierten Strahlenpaare $P$ II, $P O ; r, r^{\prime}$ einander harmonisch, und trennen in $J_{\pi x}$ die conjugierten Strahlenpaare $\Pi P, \Pi O ; \rho, \rho^{\prime}$ einander ebenfalls harmonisch, dann schneidet jede der reellen Geraden

$$
\left.\left.t_{135}=(r \rho)\left(r^{\prime} \rho^{\prime}\right)\left|, t_{246}=\right| r \rho^{\prime}\right) r^{\prime} \rho\right) \mid
$$

die $J_{p}$ und $J_{\pi}$ in dieselbe involutorische Punktreihe. Die Geraden $P \Pi, t_{13.5}, t_{246}$ sind daher die reellen Diagonalen des imaginären Vierseits $x x_{1} y y_{1}$, and das Geradenpaar $t_{135} t_{246}$ trennt das Punktepaar $P$ II harmonisch.

Ferner ist der Schnittpunkt $O$ der Geraden $t_{135} t_{246}$, wie auch der Schnittpunkt der zu $P \Pi$ conjugierten Strahlen in $J_{p}, J_{s x}$, der Pol der Geraden $P \Pi$ in Hinsicht a uf $k$. 
3. Nachdem die Strahlen $p^{\mathrm{I}} p^{\mathrm{II}} p^{\mathrm{III}} x x_{1}, \pi^{\mathrm{IV}} \pi^{\mathrm{V}} \pi^{\mathrm{VI}} y y_{1}$ äquianharmonisch sind, so hat man

$$
p^{\mathrm{I}} p^{\mathrm{II}} p^{\mathrm{III}} x x_{1} \ldots \pi\left\{\begin{array}{l}
\pi^{\mathrm{IV}} \pi^{\mathrm{V}} \pi^{\mathrm{VI}} y y_{1} \ldots \\
\pi^{\mathrm{V}} \pi^{\mathrm{VI}} \pi^{\mathrm{IV}} y y_{1} \ldots \\
\pi^{\mathrm{VI}} \pi^{\mathrm{IV}} \pi^{\mathrm{V}} y y_{1} \ldots \\
\pi^{\mathrm{IV}} \pi^{\mathrm{VI}} \pi^{\mathrm{V}} y_{1} y \ldots \\
\pi^{\mathrm{V}} \pi^{\mathrm{IV}} \pi^{\mathrm{VI}} y_{1} y \ldots \\
\pi^{\mathrm{VI}} \pi^{\mathrm{V}} \pi^{\mathrm{IV}} y_{1} y \ldots
\end{array}\right.
$$

Die Erzeugnisse der Strahlenbüschel, welche durch diese sechs projective Beziehungen bestimmt sind, seien die Kegelschnitte $k_{5} k_{3} k_{1} k_{6} k_{4} k_{2}$.

Diese Kegelschnitte gehen nicht bloß durch die Schnittpunkte der entsprechenden Strahlen der drei ersten Paare $\left(p^{i} \pi^{\top}\right)$, sondern auch durch die gegenüber liegenden Punkte $\left(p^{i} \pi^{l}\right)^{\prime}$. Ausserdem gehen die drei ersten Kegelschnitte durch die conjugiert-imaginären Punkte $(x y)\left(x_{1} y_{1}\right)$, die drei ïbrigen durch die conjugiert-imaginären Punkte $\left(x y_{1}\right)\left(x_{1} y\right)$.

Also schneiden die Kegelschnitte $k k_{1} k_{3} k_{5}$ die Gerade $t_{135}$, und die Kegelschnitte $k_{2} k_{2} k_{4} k_{6}$ die Gerade $t_{246}$, in denselben zwei conjugiert-imaginären Punkten.

Die Kegelschnitte $k_{1} k_{2} k_{3} k_{4} k_{5} k_{6}$ gehen noch bezw. durch die Eckpunkte 123456 des Paseal'schen Sechseckes.

Betrachtet man nämlich die Punkte

$$
\left(p^{\mathrm{I}} \pi^{\mathrm{IV}}\right)^{\prime} P\left(p^{\mathrm{II}} \pi^{\mathrm{V}}\right) \Pi\left(p^{\mathrm{III}} \pi^{\mathrm{VI}}\right)^{\prime} 5
$$

als die anf einander folgenden Punkte eines einfachen Sechseckes, dann sind die auf einander folgenden Seiten desselben

$$
p^{\mathrm{I}} p^{\mathrm{rI}} \pi^{\nabla} \pi^{\prime \mathrm{IV}} 4556,
$$

von welchen sich die gegenüber liegenden in der Geraden $\overline{12}$ schneiden. Aber der Kegelschnitt, welcher durch die fünf ersten angenommenen Punkte geht, ist $k_{5}$, also geht $k_{5}$ auch durch den Punkt 5. Daher:

Drei durch einen Steiner'schen Punkt gehende Pascal'sche Geraden sehneiden die dreidureh seinen Gegenpunkt gehenden Pascal'schen Geraden in neun Punkten; von diesen liegen je drei, von welchen keine $z w e i$ derselben Pascal'schen Geraden angehören, mit den Steiner'schen Gegenpunkten und mit einem Eckpunkte des Pascal'schen Sechseckes auf einen Kegelschnitt. Es gibt sechs solche Kegelschnitte; von diesen schneiden drei und ebensodie übrigen drei, den dem, Pascal'schen Sechseck umschriebenen Kegelschnitt $k$ in denselben conjugiert-imaginären 
Punkten. Der Schnittpunkt der zwei reellen Träger dieser zwei Paar conjugiert-imaginären Punkte ist der Pol der Verbindungsgeraden der Steiner'schen Gegenpunkte in Hinsicbtauf $k$.

4. Betrachten wir das Punktepaar $P \Pi$ als einen degenerierten Kegelschnitt $[P \Pi]$, die Strahlen $p^{i} p^{i} \pi^{l} \pi^{\prime l}$ als seine Tangenten. Beziehen wir diese Strahlen projectiv so auf einander, dass den Strahlen

die Strablen

$$
p^{\mathrm{I}} p^{\mathrm{II}} p^{\mathrm{III}}
$$

$$
\begin{array}{lll}
\pi^{\mathrm{VI}} \pi^{\mathrm{IV}} \pi^{\mathrm{V}} & \pi^{\mathrm{V}} \pi^{\mathrm{VI}} \pi^{\mathrm{IV}} & \pi^{\mathrm{IV}} \pi^{\mathrm{V}} \pi^{\mathrm{VI}} \\
\pi^{\mathrm{VI}} \pi^{\mathrm{V}} \pi^{\mathrm{IV}} & \pi^{\mathrm{V}} \pi^{\mathrm{IV}} \pi^{\mathrm{VI}} & \pi^{\mathrm{IV}} \pi^{\mathrm{VI}} \pi^{\mathrm{V}} \\
\left(\pi^{\mathrm{VI}} \pi^{\mathrm{IV}} \pi^{\mathrm{V}}\right)^{\prime} & \left(\pi^{\mathrm{V}} \pi^{\mathrm{VI}} \pi^{\mathrm{IV}}\right)^{\prime} & \left(\pi^{\mathrm{IV}} \pi^{\mathrm{V}} \pi^{\mathrm{VI}}\right)^{\prime} \\
\left(\pi^{\mathrm{VI}} \pi^{\mathrm{V}} \pi^{\mathrm{V}}\right)^{\prime} & \left(\pi^{\mathrm{V}} \pi^{\mathrm{IV}} \pi^{\mathrm{VI}}\right)^{\prime} & \left(\pi^{\mathrm{IV}} \pi^{\mathrm{VI}} \pi^{\mathrm{V}}\right)^{\prime}
\end{array}
$$

entsprechen, und bezeichnen wir die zwölf Projectivität-Mittelpunkte welche zu diesen zwölf projectiven Beziehungen gehören, mit

$$
\begin{array}{lll}
K_{1} & K_{3} & K_{5} \\
K_{8} & K_{4} & K_{6} \\
K_{1}^{\prime} & K_{3}{ }^{\prime} & K_{5}{ }^{\prime} \\
K_{2}{ }^{\prime} & K_{4}^{\prime} & K_{6}^{\prime}{ }^{\prime} .
\end{array}
$$

Den Strahlen $\left(p^{\mathrm{T}} p^{\mathrm{II}} p^{\mathrm{III}}\right)^{\prime}$ entsprechen bei den sechs ersten Beziehungen ebensolche mit " "versehene Strahlen $\pi^{i}$, in den übrigen Bezeichnungen ebensolche Strahlen $\pi^{i}$ aber ohne $y^{\prime} "$, wie den drei Strahen $p^{\mathrm{I}} p^{\mathrm{IL}} p^{\mathrm{III}}$.

Die gegenüberliegenden Punktepaare

$$
\begin{aligned}
& \left(p^{\mathrm{I}} \pi^{\mathrm{VI}}\right)\left(p^{\mathrm{I}} \pi^{\mathrm{VI}}\right)^{\prime},\left(p^{\mathrm{II}} \pi^{\mathrm{IV}}\right)\left(p^{\mathrm{II}} \pi^{\mathrm{IV}}\right)^{\prime}, \underset{\left.p^{\mathrm{II}} \pi^{\mathrm{V}}\right)\left(p^{\mathrm{II}} \pi^{\mathrm{V}}\right)^{\prime}}{ } \text { bilden auf } k_{1} \text { eine Involution } J_{1} \\
& \left(p^{\mathrm{I}} \pi^{\mathrm{V}}\right)\left(p^{\mathrm{I}} \pi^{\mathrm{V}}\right)^{\prime},\left(p^{\mathrm{II}} \pi^{\mathrm{VI}}\right)\left(p^{\mathrm{II}} \pi^{\mathrm{VI})^{\prime}},\left(p^{\mathrm{II}} \pi^{\mathrm{IV}}\right)\left(p^{\mathrm{III}} \pi^{\mathrm{IV}}\right)^{\prime}\right. \\
& \text { bilden auf } k_{3} \text { eine Involution } J_{3} \\
& \left(p^{\mathrm{I}} \pi^{\mathrm{IV}}\right)\left(p^{\mathrm{I}} \pi^{\mathrm{IV}}\right)^{\prime},\left(p^{\mathrm{II}} \pi^{\mathrm{V}}\right)\left(p^{\mathrm{II}} \pi^{\mathrm{V}}\right)^{\prime},\left(p^{\mathrm{III}} \pi^{\mathrm{VI}}\right)\left(p^{\mathrm{III}} \pi^{\mathrm{VI}}\right)^{\prime} \\
& \text { bilden auf } k_{5} \text { eine involution } J_{5} \\
& \left(p^{\mathrm{I}} \pi^{\mathrm{VI}}\right)\left(p^{\mathrm{I}} \pi^{\mathrm{VI}}\right)^{\prime},\left(p^{\mathrm{II}} \pi^{\mathrm{V}}\right)\left(p^{\mathrm{II}} \pi^{\mathrm{V}}\right)^{\prime},\left(p^{\mathrm{II}} \pi^{\mathrm{IV}}\right)\left(p^{\mathrm{III}} \pi^{\mathrm{IV}}\right)^{\prime} \\
& \text { bilden auf } k_{2} \text { eine Involution } J_{2} \\
& \left(p^{\mathrm{I}} \pi^{\mathrm{V}}\right)\left(p^{\mathrm{I}} \pi^{\mathrm{V}}\right)^{\prime},\left(p^{\mathrm{II}} \pi^{\mathrm{IV}}\right)\left(p^{\mathrm{II}} \pi^{\mathrm{IV}}\right)^{\prime},\left(p^{\mathrm{III}} \pi^{\mathrm{VI}}\right)\left(p^{\mathrm{III}} \pi^{\mathrm{VI}}\right)^{\prime} \\
& \text { bilden auf } k_{4} \text { eine Involution } J_{4} \\
& \left(p^{\mathrm{I}} \pi^{\mathrm{IV}}\right)\left(p^{\mathrm{I}} \pi^{\mathrm{IV}}\right)^{\prime},\left(p^{\mathrm{II}} \pi^{\mathrm{VI}}\right)\left(p^{\mathrm{II}} \pi^{\mathrm{VI}}\right)^{\prime},\left(p^{\mathrm{III}} \pi^{\mathrm{V}}\right)\left(p^{\mathrm{III}} \pi^{\mathrm{V}}\right)^{\prime} \\
& \text { bilden auf } k_{6} \text { eine Involution } J_{6},
\end{aligned}
$$

deren Involutions-Mittelpunkte $K_{1}^{\prime} K_{3}{ }^{\prime} K_{5}^{\prime} K_{2}{ }^{\prime} K_{4}{ }^{\prime} K_{6}{ }^{\prime}$ sind.

Die Pole der Verbindungsgeraden der gegentiber liegenden Punkte in Hinsicht auf die Kegelschnitte $k_{i}$, auf welchen sie liegen, 
ferner die Schnittpunkte dieser Verbindungsgeraden mit den Involutions-Axen der durch die gegenüber liegenden Punkte als conjugierten Punkten bestimmten Involutionen $J_{i}$ sind bezw.

$$
\begin{array}{ll}
K_{2} K_{4} K_{6} & K_{2}^{\prime} K_{4}^{\prime} K_{6}^{\prime} \\
K_{4} K_{6} K_{2} & K_{4}^{\prime} K_{6}^{\prime} K_{2}^{\prime} \\
K_{6} K_{2} K_{4} & K_{6}^{\prime} K_{2}^{\prime} K_{4}^{\prime} \\
K_{1} K_{5} K_{3} & K_{1}^{\prime} K_{5}^{\prime} K_{3}^{\prime} \\
K_{3} K_{1} K_{5} & K_{3}^{\prime} K_{1}^{\prime} K_{5}^{\prime} \\
K_{5} K_{3} K_{1} & K_{5}^{\prime} K_{3}^{\prime} K_{1}^{\prime} .
\end{array}
$$

So z. B. ist der Pol der Verbindungsgeraden der gegenüber liegenden Punkte $\left(p^{\mathrm{I}} \pi^{\mathrm{VI}}\right)\left(p^{\mathrm{I}} \pi^{\mathrm{VI}}\right)^{\prime}$ in Hinsicht auf $k$, der Schnittpunkt $K_{2}$ der Geraden $\left|\left(p^{\mathrm{II}} \pi^{\mathrm{IV}}\right)\left(p^{\mathrm{III}} \pi^{\mathrm{V}}\right)\right|,\left|\left(p^{\mathrm{II}} \pi^{\mathrm{IV}}\right)^{\prime}\left(p^{\mathrm{III}} \pi^{\mathrm{V}}\right)^{\prime}\right|$.

Ferner schneidet die Gerade $\left|\left(p^{\mathrm{I}} \pi^{\mathrm{VI}}\right)\left(p^{\mathrm{T}} \pi^{\mathrm{VI}}\right)^{\prime}\right|$, welche die conjugierten Punkte $\left(p^{\mathrm{I}} \pi^{\mathrm{VI}}\right),\left(p^{\mathrm{I}} \pi^{\mathrm{VI}}\right)^{\prime}$ der Involution $J_{1}$ verbindet, die.zu dieser Involution gehörige Involutions-Axe im Schnittpunkte $K_{2}^{\prime}$ der Geraden $\left|\left(p^{\mathrm{II}} \pi^{\mathrm{IV}}\right)\left(p^{\mathrm{III}} \pi^{\mathrm{V}}\right)^{\prime}\right|,\left|\left(p^{\mathrm{III}} \pi^{\mathrm{V}}\right)\left(p^{\mathrm{II}} \pi^{\mathrm{IV}}\right)^{\prime}\right|$.

Die conjugierten Punktepaare $K_{2} K_{2}^{\prime}, K_{4} K_{4}^{\prime}, K_{6} K_{6}^{\prime}$ bilden eine Involution $J_{135}$, die conjugierten Punktepaare $K_{1} K_{1}^{\prime}, K_{3} K_{3}^{\prime}, K_{5} K_{5}^{\prime}$ bilden eine Involution $J_{246}$; die erste Involution hat mit $J_{1} J_{3} J_{5}$, die zweite Involution hat mit $J_{2} J_{4} J_{6}$ gemeinsame conjugiert-imaginäre Doppelpunkte, welche die gemeinsamen Schnittpunkte $(x y)\left(x_{1} y_{1}\right),\left(x y_{1}\right)\left(x_{1} y\right)$ der Kegelchnitte $k_{1} k_{3} k_{5}$, beziehungsweise $k_{2} k_{4} k_{6}$ sind. Daher sind die Träger der Involutionen $J_{135}, J_{246}$ die früheren Geraden $t_{135}, t_{246}$, und es ist

$$
\begin{aligned}
& t_{135} \equiv K_{2} K_{4} K_{6} K_{2}^{\prime} K_{4}^{\prime} K_{6}^{\prime} \mid \\
& t_{246} \equiv K_{1} K_{3} K_{5} K_{1}^{\prime} K_{3}^{\prime} K_{5}^{\prime} \mid .
\end{aligned}
$$

Die nean Verbindungsgeraden der Punkte $K_{1}^{\prime} K_{3}^{\prime} K_{5}^{\prime}$ mit den Punkten $K_{2}^{\prime} K_{4}^{\prime} K_{6}^{\prime}$ sind zugleich die Verbindungsgeraden der neun Paar gegenüber liegenden Punkte $\left(p^{i} \pi^{l}\right)\left(p^{i} \pi^{l}\right)^{\prime}$, und auf jeder dieser Geraden werden die gegenüber liegenden Punkte von den zwei Punkten $K_{i}^{\prime}$ harmonisch getrennt. Es strahlen aber noch aus jedem der sechs Punkte $K_{i}^{\prime}$ auch solche sechs Geraden aus, die zwei Punkte wie $\left(p^{i} \pi^{l}\right)\left(p^{j} \pi^{m}\right)^{\prime}$ tragen, welche aber nicht gegenüber liegende sind. So z. B. trennen die lunktepare $K_{1}^{\prime} K_{2}^{\prime}, K_{1}^{\prime} K_{4}^{\prime}$, $K_{1}^{\prime} K_{6}^{\prime}$, beziehungsweise die Punktepaare $\left.\left(p^{\mathrm{I}} \pi^{\mathrm{VI}}\right)\left(p^{\mathrm{I}} \pi^{1}\right)^{\prime}\right),\left(p^{\mathrm{II}} \pi^{\mathrm{IV}}\right)$ $\left(p^{\mathrm{II}} \pi^{\mathrm{IV}}\right)^{\prime},\left(p^{\mathrm{II}} \pi^{\mathrm{V}}\right)\left(p^{\mathrm{III}} \pi^{\mathrm{V}}\right)^{\prime}$ harmonisch, und aus $K_{1}^{\prime}$ strahlen aus die Geraden $\left(p^{\mathrm{I}} \pi^{\mathrm{V}}\right)\left(p^{\mathrm{II}} \pi^{\mathrm{VI}}\right)^{\prime},\left(p^{\mathrm{II}} \pi^{\mathrm{VI}}\right)\left(p^{\mathrm{I}} \pi^{\mathrm{IV}}\right)^{\prime},\left(p^{\mathrm{III}} \pi^{1 \mathrm{~V}}\right)\left(p^{\mathrm{II}} \pi^{\mathrm{V}}\right)^{\prime},\left(p^{\mathrm{I}} \pi^{\mathrm{IV}}\right)$ $\left(p^{\mathrm{II}} \pi^{\mathrm{VI}}\right)^{\prime},\left(p^{\mathrm{II}} \pi^{\mathrm{V}}\right)\left(p^{\mathrm{II}} \pi^{\mathrm{IV}}\right)^{\prime},\left(p^{\mathrm{III}} \pi^{\mathrm{VI}}\right)\left(p^{\mathrm{I}} \cdot \pi^{\mathrm{V}}\right)^{\prime}$. 
Die Pole der Geraden $P I I$ in Hinsicht auf die Kegelschnitte $k_{1} k_{2} \ldots k_{6}$ sind die Punkte $K_{1} K_{2} \ldots K_{6}$, denn es schneiden sich 2. B. die Diagonale $\left(p^{\mathrm{I}} \pi^{\mathrm{IV}}\right)\left(p^{\mathrm{II}} \pi^{\mathrm{VI}}\right),\left(p^{\mathrm{I}} \pi^{\mathrm{V}}\right)\left(p^{\mathrm{III}} \pi^{\mathrm{VI}}\right)$ der dem Kegelschnitt $k_{1}$ einbeschriebenen Vierecke $P \Pi\left(p^{\mathrm{I}} \pi^{\mathrm{VI}}\right)\left(p^{\mathrm{II}} \pi^{\mathrm{IV}}\right)$, $P \Pi\left(p^{\mathrm{I}} \pi^{\mathrm{VI}}\right)\left(p^{\mathrm{II}} \pi^{\mathrm{V}}\right)$, im Pole der Geraden $P I I$ in Hinsicht auf $k_{1}$.

Daraus folgt ferner, dass der Pol der Geraden $t_{135}$ in Hinsicht auf die Kegelschnitte $k_{2} k_{4} k_{6}$, der Punkt $\left(t_{246}, \mid P[||)\right.$, und der Pol der Geraden $t_{246}$ in Hinsicht auf die Kegelschnitte $k_{1} k_{3} k_{5}$ der Punkt $\left(t_{135}, \mid P[\mid)\right.$ ist. Denn die Punkte $K_{1}^{\prime}, K_{1}$ sind die Pole der Geraden $t_{135}, P \Pi \mid$ in Hinsicht auf $k_{1}$, also ist der Punkt $\left(t_{135},|P I I|\right)$ der Pol der Geraden $t_{246}=K_{1} K_{1}^{\prime}$.

Die erhaltenen Resultate können wir als Fortsetzung der früheren Sätze in Folgendem zusanmenfassen:

Die a uf jeden der Kegelschnitte $k_{1} k_{3} k_{5} k_{2} k_{4} k_{6}$ liegenden drei Punktepare $\left(p^{i} \pi^{2}\right)\left(p^{i} \pi^{l}\right)^{\prime}$ sind conjugierte Punktevon Involutionen $J_{1} J_{3} J_{5} J_{2} J_{4} J_{6}$; ihre InvolutionsMittelpunkte sind $K_{1}^{\prime} K_{3}^{\prime} K_{5}^{\prime} K_{2}^{\prime} K_{4}^{\prime} K_{6}^{\prime}$. D ie gemeinsame Involutions. Axe der Involutionen $J_{1} J_{3} J_{5}$ ist die durch die Punkte $K_{2}^{\prime} K_{4}^{\prime} K_{6}^{\prime}$ gehende Gerade $t_{135}$, und die gemeinsame Involutions-Axe der Involutionen ist die durch die Punkte $K_{1}^{\prime} K_{3}^{\prime} K_{5}^{\prime}$ gehende Gerade $t_{246}$.

Die Pole $K_{1} K_{3} K_{5}$ und $K_{2} K_{4} K_{6}$ der Geraden PII in Hinsicht auf die Kegelschnitte $k_{1} k_{3} k_{5}$, beziehungsweise $k_{2} k_{4} k_{6}$, liegen aufder Geraden $t_{246}$, beziehungsweise $t_{135} ; K_{1} K_{1}^{\prime}, K_{3} K_{3}^{\prime}, K_{5} K_{5}^{\prime}$, so wie $K_{2} K_{2}^{\prime}, K_{4} K_{4}^{\prime}, K_{6} K_{6}^{\prime}$ sind conjugierte Punkte von zwei lnvolutionen $J_{135}$, $J_{246}$. Diegemeinsamen Doppelpunkte der Involutionen $J_{1} J_{3} J_{5} J_{13 \tilde{b}}$, und $J_{2} J_{4} J_{6} J_{246}$ sind diefrüheren Punkte $(x y)\left(x_{1} y_{1}\right),\left(x y_{1}\right)\left(x_{1} y\right)$, welche mit jedem der drei auf den Kegelschniten $k_{1} k_{2} \ldots k_{6}$ liegenden Punkten $\left(p^{i} \pi^{i}\right)$, wie auch $\left(p^{i} \pi^{\prime}\right)^{\prime}$, und mit den auf den Geraden $t_{135}, t_{246}$ liegenden Punkte $K$, wie auch $K_{i}^{\prime}$, äquianharmonisch sind.

A us jedem der sechs Punkte $K_{i}^{\prime}$ Strahlen neun Gerade aus, welche einen Punkt $\left(p^{i} \pi^{\prime}\right)$ und einen Punkt $\left(p^{j} \pi^{\prime \prime \prime}\right)^{\prime}$ tragen; a uf dreien dieser Geraden sind die genannten Punkte gegenüber liegende, und trennendie zwei auf diesen Geraden befindliehen Punkte $K^{\prime}$ harmoniseb.

5. Wir müssen hier folgenden Hilfssatz über Kegelschnitte beweisen: "Wenn zwei Kegelschnitte zwei gegenüber liegende Eck. punkte eines Vierseits mit einander gemein haben, und jeder auch noch durch zwei außerhalb des anderen liegende Eckpunkte des Vierseits geht, so schneiden sich die Geraden, welche den einen. 
gleichviel welchen von den beiden Kegelschnitten in jenen gemeinschaftlichen Punkten herühren, und die Geraden, welche den anderen in den übrigen gemeinschaftlichen Punkten der beiden Kegelschnitte sich anschmiegen, in einem und demselben Punkte" (Staudt 1. c.).

Beweis: Nach einem bekannten Satze von $S$ teiner sind die acht Geraden, welche zwei Kegelschnitte in ihren vier Schnittpunkten berühren, die Tangenten eines Kegelschnittes. Schneiden sich also drei dieser Tangenten in einem Punkte $U$, so degeneriert dieser Kegelschnitt in ein Punktepaar $U V$. Die übrigen fünf Tangenten können aber nicht alle durch den anderen Punkt $V$ gehen, da sie aus den vier Schnittpunkten ausstrahlen und also zwei derselben keinen zweiten gemeinsamen Punkt baben können; es müssen daher in dem betrachteten Falle vier der Tangenten durch einen Punkt $U$ und vier durch einen Punkt $V$ gehen.

Um aber zu zeigen, dass die Tangenten in drei Schnittpunkten der im Satze angegebenen Kegelschnitte sich in einem Punkt schneiden, bezeichnen wir die Kegelschnitte, welche durch die Gegeneckpunkte $B B_{1}, C C_{1}$ und $A A_{1}, C C_{1}$ eines Vierseits $A A_{1} B B_{1} C C_{1}$ gehen, mit $k_{a}, k_{b}$; diese hahen außer $C C_{1}$ noch die Schnittpunkte $D D_{1}$.

Der Pol $U$ der Geraden $\left|C C_{1}\right|$ in Hinsicht auf $k_{a}$ ist der Schnittpunkt der zu $\left|C C_{1}\right|$ conjugierten Polaren $\left|A A_{1}\right|, t=$ $=\mid\left(C_{1} B_{1}, C D\right)\left(C B_{1}, C_{1} D\right)$, wie dies aus den Vierecken $C C_{1} B B_{1}$, $C C_{1} B_{1} D$ ersichtlich. Die Gerade $t$ ist aber die Pascal'sche Gerade der dem Kegelschnitt $k_{b}$ einbeschriebenen Sechsecke $C A_{1} A C_{1} D D$, (denn $C B_{1} \equiv C A_{1}, C_{1} B \equiv C_{1} A$ ), also berührt die durch den Punkt $U$ gehende Gerade $t$ den Kegelschnitt $k_{b}$ im Schnittpnnkte $D$.

Wir bemerken noch, dass der Pol der Geraden $B B_{1}$ in Hinsicht auf $k_{1}$, welcher den Punkt $U$ vom Punktepaare $A A_{1}$ har" monisch trennt, auf der Geraden $D D_{1}$ liegt.

6. Wir kehren wieder zu der früheren Configuration zurück. Der Kegelschnitt $k$ geht durch die Eckpunkte $(x y)\left(x_{1} y_{1}\right)\left(x y_{1}\right)\left(x_{1} y\right)$, die Kegelschnitte $k_{1} k_{3} k_{5}$ gehen durch die Eckpunkte PII $(x y)\left(x_{1} y_{1}\right)$, und die Kegelschnitte $k_{2} k_{4} k_{6}$ gehen durch die Eckpunkte PII $\left(x y_{1}\right)\left(x_{1} y\right)$ des Vierseits $x y x_{1} y_{1}$. Daher hat der Kegelschnitt $k$ ge. genüber den Kegelschnitten $k_{1} k_{2} \ldots k_{6}$, und $k_{1} k_{3} k_{5}$ gegenüber $k_{2} k_{4} k_{6}$ dieselbe Lage, wie die Kegelschnitte $k_{a}, k_{b}$ der früheren Nummer.

Der Kegelschnitt $k$ schneidet schon die Kegelschnitte $k_{1} k_{2} \ldots k_{6}$ in den Punkten $12 \ldots 6$, and wir wollen die vierten Schnittpunkte mit $1^{\prime} 2^{\prime} \ldots 6^{\prime}$ bezeichnen.

Also gehen die Tangenten des Kegelschnittes $k$ in den Punkte-

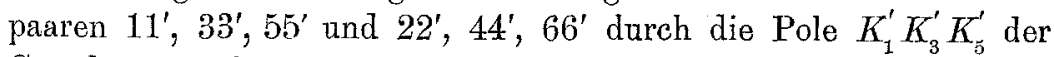
Geraden $t_{135}$ in Hinsicht auf $k_{1} k_{3} k_{5}$, beziehungsweise durch die Pole $K_{2}^{\prime} K_{4}^{\prime} K_{6}^{\prime}$ der Geraden $t_{246}$ in Hinsicht auf $k_{2} k_{4} k_{6}$. Außer 
diesem schneiden die Geraden $11^{\prime}, 33^{\prime}, 55^{\prime}$, die Gerade $t_{246}$ and die Geraden $22^{\prime}, 44^{\prime}, 66^{\prime}$, die Gerade $t_{135}$ in denjenigen Punkten $K_{1} K_{3} K_{5}$, beziehungsweise $K_{2} K_{4} K_{6}$, welche das Punktepaar $\left(x y_{1}\right)\left(x_{1} y\right)$ beziehungsweise das Punktepaar $(x y)\left(x_{1} y_{1}\right)$ von den Punkten $K_{1}^{\prime} K_{3}^{\prime} K_{5}^{\prime}$, beziehungsweise $K_{2}^{\prime} K_{4}^{\prime} K_{6}^{\prime}$ harmonisch trennen. Die Tangenten des Kegelschnitts $k$ in den Punkten $12 \ldots 6$ gehen also durch die Punkte $K_{1}^{\prime} K_{2}^{\prime} \ldots K_{6}^{\prime}$.

Anderseits schneiden sich die Tangenten von $k_{1} k_{3} k_{5}$ und

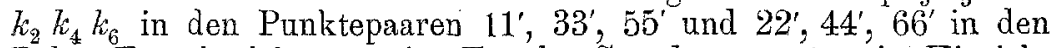
Polen $T_{135}$, beziehungsweise $T_{246}$ der Geraden $t_{135}, t_{246}$ in Hiusicht auf $k$; ferner trennt der gemeinsame Schnittpunkt der Geraden $11^{\prime}, 33^{\prime}, 55^{\prime}$, so wie der Geraden $22^{\prime}, 44^{\prime}, 66^{\prime}$ die Punkte $T_{135}$, beziehungsweise $T_{246}$ vom Punktepaare $P$ [I harmonisch. Aber diese Schnittpunkte sind beziehungsweise $T_{246}, T_{135}$, denn die Pole der drei ersten Geraden in Hinsicht auf $k$ sind die auf $t_{246}$ liegende Punkte $K_{1}^{\prime} K_{3}^{\prime} K_{i}^{\prime}$, und die Pole der drei anderen Geraden in Hinsicht auf $k$ sind die auf $t_{135}$ liegende Punkte $K_{2}^{\prime} K_{4}^{\prime} K_{6}^{\prime}$.

Nachdem die Tangenten von $k$ in den Punkten 135 und $1^{\prime} 3$ '5' die Gerade $t_{246}$ in den Punkten $K_{1}^{\prime} K_{3}^{\prime} K_{5}^{\prime}$ schneiden und $K_{1}^{\prime} K_{3}^{\prime} K_{5}^{\prime}\left(x y_{1}\right)\left(x_{1} y\right)$ aquianharmonische Punkte sind, so werden auch $135\left(x y_{1}\right)\left(x y_{1}\right)$ und $1^{\prime} 3^{\prime} 5^{\prime}\left(x y_{1}\right)\left(x_{1} y\right)$ äquianharmonisch sein, und es gehen also die Geradenpare $35,3^{\prime} 5^{\prime} ; 51,5^{\prime} 1^{\prime} ; 13,1^{\prime} 3^{\prime}$ durch die Punkte $K_{1}^{\prime} K_{3}^{\prime} K_{5}^{\prime}$. Aus demselben Grunde sind die Punkte $246(x y)\left(x_{1} y_{1}\right)$. $2^{\prime} 4^{\prime} 6^{\prime}(x y)\left(x_{1} y_{1}\right)$ äquianharmoniseh und die Geradenpare $46,4^{\prime} 6^{\prime}$; $62,6^{\prime} 2^{\prime} ; 24,2^{\prime} 4^{\prime}$ schneiden sich in den Punkten $K_{2}^{\prime} K_{4}^{\prime} K_{6}^{\prime}$.

In Anbetracht dessen, dass die Punkte $1^{\prime} 2^{\prime} \ldots 6^{\prime}$ gegenüber den Kegelschnitten $k k_{1} k_{2} \ldots k_{6}$, den Geraden $t_{135} t_{246}$, den Punkten $P \amalg I T_{135} T_{246} K_{1} K_{2} \ldots K_{6} K_{1}^{\prime} K_{2}^{\prime} \ldots K_{6}^{\prime}$ dieselbe Lage haben, wie die Punkte $12 \ldots 6$, werden die sechs Pascal'sche Gerade $p^{\mathrm{I}} p^{\mathrm{II}} \ldots p^{\mathrm{VI}}$, auch Pascal'sche Geraden des Sechseckes 1' 2' $3^{\prime} 4^{\prime} 5^{\prime} 6^{\prime}$ sein.

Daher: Die Kegelschnitte $k_{1} k_{2} \ldots k_{6}$ schneiden $k$ in den reellen Punktepaaren $11^{\prime} 22^{\prime}, \ldots, 66^{\prime}$, deren Verbindungsgeraden durch die Punkte $K_{1} K_{2} \ldots K_{6}$ gehen. Die Tangentenpare in diesen Punkteparen zu dem Kegelschnitt $k$ schneiden sich in den Punkten $K_{1}^{\prime} K_{2}^{\prime} \ldots K_{6}^{\prime}$, und za den Kegelsehnitten $k_{1} k_{3} k_{5}, k_{2} k_{4} k_{6}$ in den Polen $T_{135}, T_{246}$ der Geraden $t_{135}, t_{246}$ in Hinsichtauf $k$. Diese Pole $T_{135}, T_{246}$ trennen $P$ harmonisch. Die Geraden $p^{\mathrm{I}} p^{\mathbf{I I}} \ldots \pi^{\mathrm{VI}}$ sind $\mathrm{Pascal}$ sche Geraden des Sechseckes $1^{\prime} 2^{\prime} 3^{\prime} 4^{\prime} 5^{\prime} 6^{\prime}$.

7. Man sieht aus dem Früheren, dass die Geraden $\left(p^{\mathrm{I}} p^{\mathrm{II}} p^{\mathrm{II}}\right.$. $\left.\pi^{\mathrm{IV}} \pi^{\mathrm{V}} \pi^{\mathrm{VI}}\right)^{\prime}$ die Kegelschnitte $k_{1} k_{2} \ldots k_{6}$, die Geraden $t_{135}, t_{246}$ unc die auf diesen liegende Punkte $K_{2} K_{4} K_{6} K_{2}^{\prime} K_{4}^{\prime} K_{6}^{\prime}(x y)\left(x_{1} y_{2}\right)$ : $K_{1} K_{3} K_{5} K_{1}^{\prime} K_{3}^{\prime} K_{5}^{\prime}\left(x y_{1}\right)\left(x_{1} y\right)$, durch die sechs sich zu dreien ir 
den Punkten $P, I I$ schneidenden Geraden $p^{\mathrm{I}} p^{\mathrm{II}} p^{\mathrm{III}}, \pi^{\mathrm{IV}} \pi^{\mathrm{V}} \pi^{\mathrm{rI}}$ bestimmt sind. In Anbetracht dessen, dass die neun Verbindungsgeraden der Eckpunkte des Dreieckes 135 mit den Eckpunkten des Dreieckes 246 durch die ueun Punkte $\left(p^{i} \pi^{l}\right)^{\prime}$, und ihre sechs Seiten durch die Punkte $K_{1}^{\prime} K_{3}^{\prime} K_{5}^{\prime}, K_{2}^{\prime} K_{4}^{\prime} K_{6}^{\prime}$ gehen, kann man, wenn die Geraden $p^{\mathrm{I}} p^{\mathrm{II}} p^{\mathrm{III}} \pi^{\mathrm{IV}} \pi^{\mathrm{V}} \pi^{\mathrm{VI}}$ und ein Eckpunkt des Sechseckes $12 \ldots 6$ gegeben ist, die übrigen fünf Eckpunkte, den durch dieselben gehenden Kegelschnitt $k$, und die neun Punkte 1'2' ..6 6', construieren.

Beschreibt der Punkt 1 auf $k_{1}$ eine Reihe $R_{1}$, so werden die Punkte $23 \ldots 6$ auf den Kegelschnitten $k_{2} k_{3} \ldots k_{6}$ mit $R_{1}$ projective Reihen $R_{2}, R_{3}, \ldots, R_{6}$ beschreiben, in welchen der Punkt $P$ und der Punkt II entsprechend ist. Diese Punktreihen sind zugleich projectiv mit denjenigen neun Strahlenbüscheln, deren Mittelpunkte die Punkte $\left(p^{i} \pi^{2}\right)^{\prime}$ sind and welche die Punktreihen $R_{1}, R_{3}, R_{5}$, in die Punktreihen $R_{2}, R_{4}, R_{6}$ projiciern. Jeder der Kegelschnitte $k_{1} \cdot k_{2} \ldots k_{6}$ ist das Erzeugnis von solchen drei dieser Strahlenbuischel, deren Mittelpunkte auf dem betreffenden Kegelschnitt liegen. Hingegen ist das Krzeugnis von zwei solchen Strahlenbüscheln, deren Mittelpunkte mit dem Punkte $P$ oder mit dem Punkte II auf einer Geraden $\left(p^{i}\right.$ oder $\left.\pi^{\prime l}\right)$ liegen, eine Gerade $\pi^{m}$ oder $p^{j}$, welche von den Mittelpunkten der Strahlenbüschel durch den auf dem gemeinsamen Strable der Büschel liegenden dritten Mittelpunkt der Büschel harmonisch getrennt ist.

Es ist z. B. das Erzeugnis der Büschel mit den Mittelpunkten $\left(p^{I} \pi^{\mathrm{TV}}\right)^{\prime},\left(p^{\mathrm{T}} \pi^{\mathrm{V}}\right)^{\prime}$, bei der obigen projectiven Beziehung die Gerade $\pi^{\mathrm{VI}}$, da sich die entsprechenden Strahlenpaare $\pi^{\prime 1 \mathrm{~V}}, \pi^{\prime \mathrm{V}} ; 56,34$, auf der Geraden $\pi^{\mathrm{VI}}$ schneiden.

Nach diesem können wir die zwei in der Einleitung erwähnten Sätze von Staudt mit der hier benützten Bezeichnung aufschreiben; sie lauten:

„11. Durch sechs in einerlei Ebene liegende Geraden, von welchen drei $p^{\mathrm{I}} p^{\Pi} p^{\mathrm{TII}}$ in einem Punkte $P$, die übrigen drei $\pi^{I V} \pi^{V} \pi^{\mathrm{VI}}$ in einem anderen Punkte II sich schneiden, sind im allgemeinen sechs Curven $k_{1} k_{3} k_{5} k_{2} k_{4} k_{6}$ zweiter Ordnung bestimmt, welche projectivisch so auf einander bezogen werden können, dass auch je sechs homologe Punkte 1, 3, 5, 2, 4, 6 derselben in einer Curve $k$ zweiter Ordnung liegen und überdies die Pascal'schen Geraden der Sechsecke

$$
\begin{array}{lll}
163254, & 123456, & 143652 \\
163452, & 123654, & 143256
\end{array}
$$

mit den gegebenen Geraden zusammenfallen.

Jede der sechs festen Curven geht durch die beiden Punkte $P$, II und durch drei von den neun Punkten $p \pi$, in welchen nämlich die drei Geraden $p^{\mathrm{I}} p^{\mathrm{II}} p^{\mathrm{II} 1}$ von den drei Geraden $\pi^{\mathrm{IV}} \pi^{\mathrm{V}} \pi^{\mathrm{VI}}$ geschnitten werden, und zwar geht 


$$
\begin{aligned}
& k_{1} \text { durch } p^{\mathrm{I}} \pi^{\mathrm{VI}}, p^{\mathrm{II}} \pi^{\mathrm{VI}}, p^{\mathrm{III}} \pi^{\mathrm{V}} \\
& k_{3} \quad \Rightarrow \quad p^{\mathrm{I}} \pi^{\mathrm{V}}, p^{\mathrm{II}} \pi^{\mathrm{VI}}, p^{\mathrm{III}} \pi^{\mathrm{IV}} \\
& k_{5} \quad n \quad p^{\mathrm{I}} \pi^{\mathrm{IV}}, p^{\mathrm{II}} \pi^{\mathrm{V}}, p^{\mathrm{III}} \pi^{\mathrm{VI}} \\
& k_{2} \quad \# \quad p^{\mathrm{I}} \pi^{\mathrm{VI}}, p^{\mathrm{II}} \pi^{\mathrm{V}}, p^{\mathrm{III}} \pi^{\mathrm{IV}} \\
& k_{4} \quad \because \quad p^{\mathrm{I}} \pi^{\mathrm{V}}, p^{\mathrm{II}} \pi^{\mathrm{IV}}, p^{\mathrm{III}} \pi^{\mathrm{VI}} \\
& k_{6} \quad \Rightarrow \quad p^{\mathrm{I}} \pi^{\mathrm{IV}}, p^{\mathrm{II}} \pi^{\mathrm{VI}}, p^{\mathrm{III}} \pi^{\mathrm{V}} \text {. }
\end{aligned}
$$

Legt man durch den Punkt $P$ die Geraden $p^{\prime I} p^{\prime \text { II }} p^{\text {III }}$ und durch den Punkt II die Geraden $\pi^{\prime \mathrm{IV}} \pi^{\prime \mathrm{V}} \pi^{\prime \mathrm{VI}}$ so dass $p^{\mathrm{II}} p^{\mathrm{III}} p^{\mathrm{I}} p^{\text {II }}$, $p^{\mathrm{III}} p^{\mathrm{I}} p^{\mathrm{II}} p^{\prime \mathrm{II}}, p^{\mathrm{I}} p^{\mathrm{II}} p^{\mathrm{III}} p^{\prime \mathrm{III}}, \pi^{\mathrm{V}} \pi^{\mathrm{VI}} \pi^{\mathrm{IV}} \pi^{\prime \mathrm{IV}}, \pi^{\mathrm{VI}} \pi^{\mathrm{IV}} \pi^{\mathrm{V}} \pi^{\mathrm{V}}, \pi^{\mathrm{IV}} \pi^{\mathrm{V}} \pi^{\mathrm{VI}} \pi^{\prime \mathrm{VI}}$ harmonische Würfe sind, so gebt nun auch jede der sechs festen Curven durch drei der neun Punkte $p^{\prime} \pi^{\prime l}$ so dass jede der drei Curven $k_{1} k_{3} k_{5}$, jede der drei Curven $k_{2} k_{4} k_{6}$ in den Punkten $P$, Il und in zwei einander gegenüber liegenden von den achtzehn Punkten $p \pi, p^{\prime} \pi^{\prime}$ schneidet, wenn man nämlich dem Punkte $p^{i} \pi^{l}$ den Punkt $p^{i} \pi^{\prime l}$ gegenüber liegend nennt.

Der Strahlenbüschel $P$ enthält zwei Strablen $x, x_{1}$, der Strahlenbüschel II zwei Strahlen $y, y_{1}$, so dass die Gebilde $p^{\mathrm{I}} p^{\mathrm{II}} p^{\mathrm{II}} x x_{1}, p^{\mathrm{II}} p^{\mathrm{III}} p^{\mathrm{I}} x x_{1}, \pi^{\mathrm{IV}} \pi^{\mathrm{V}} \pi^{\mathrm{VI}} y y_{1}$ zu einander, mithin zu denselben auch die Gebilde $p^{\mathrm{III}} p^{\mathrm{I}} p^{\mathrm{II}} x x_{1}, p^{\mathrm{I}} p^{\mathrm{III}} p^{\mathrm{II}} x_{1} x$ u. s. w. projectivisch sind, daher die drei Curven $k_{1} k_{3} k_{5}$ in den vier Punkten $P$, II $x y, x_{1} y_{1}$, die drei Curven $k_{2} k_{3} k_{4}$ aber in den vier Punkten $P$, II, $x y_{1}, x_{1} y$ sich schneiden.

Die Brianchon'schen Punkte $K_{1}, K_{3}, K_{5}$ der Sechsseiten $p^{\mathrm{I}} \pi^{\mathrm{V}}$ $p^{\mathrm{II}} \pi^{\mathrm{VI}} p^{\mathrm{III}} \pi^{\mathrm{IV}}, p^{\mathrm{I}} \pi^{\mathrm{IV}} p^{\mathrm{II}} \pi^{\mathrm{V}} p^{\mathrm{III}} \pi^{\mathrm{VI}}, p^{\mathrm{I}} \pi^{\mathrm{VI}} p^{\mathrm{II}} \pi^{\mathrm{IV}} p^{\mathrm{III}} \pi^{\mathrm{V}}$ sind die Pole der Geraden $P \Pi$ in Hinsicht auf die Curven $k_{1}, k_{3}, k_{5}$ und liegen daher in der Geraden $t_{246}$, welche den Punkt $x y_{1}$ mit dem Punkte $x_{1} y$ verbindet. Die Brianchon'schen Punkte $K_{2}, K_{4}, K_{6}$ der Sechseiten $p^{\mathrm{I}} \pi^{\mathrm{IV}} p^{\mathrm{II}} \pi^{\mathrm{VI}} p^{\mathrm{III}} \pi^{\mathrm{V}}, p^{\mathrm{T}} \pi^{\mathrm{VI}} p^{\mathrm{II}} \pi^{\mathrm{V}} p^{\mathrm{III}} \pi^{\mathrm{IV}} p^{\mathrm{I}} \pi^{\mathrm{V}} p^{\mathrm{II}} \pi^{\mathrm{IV}} p^{\mathrm{III}} \pi^{\mathrm{VI}}$ sind die Pole der Geraden PII in Hinsicht auf die Curven $k_{2} k_{4} k_{6}$ und liegen daher in der Geraden $t_{135}$, welche den Punkt $x y$ mit dem Punkte $x_{1} y_{1}$ verbindet.

In der Geraden $t_{246}$ liegen auch $K_{1}^{\prime}, K_{3}^{\prime}, K_{5}^{\prime}$ welche die Pole der Geraden $t_{135}$ in Hinsicht auf die Curven $k_{1}, k_{3}, k_{5}$ sind. Ebenso liegen in der Geraden $t_{135}$ die Punkte $K_{2}^{\prime}, K_{4}^{\prime}, K_{6}^{\prime}$, welche die Pole der Geraden $t_{246}$ in Hinsicht auf die Curven $k_{2} k_{4} k_{6}$ sind Jede der neun Geraden, welche die Punkte $K_{1}^{\prime}, K_{3}^{\prime}, K_{4}^{\prime}$ mit den Punkten $K_{2}^{\prime}, K_{4}^{\prime}, K_{6}^{\prime}$ verbindet, geht durch zwei einander gegeniiber liegende von den achtzehn Punkten $p \pi, p^{\prime} \pi^{\prime}$. Durch jeden der Punkte $K_{1}^{\prime}, K_{2}^{\prime}, \ldots, K_{6}^{\prime}$ gehen aber sechs von den sechsunddreißig Geraden, deren jede nur eine der Curven $k_{1}, k_{2}, \ldots, k_{6}$ in einem der Punkte $p \pi$ und zugleich in einem der Punkte $p^{\prime} \pi^{\prime}$ schneidet.

Die neun Strahlenbüschel, welche die Punkte $p^{*} \pi^{\prime}$ zu Mittelpunkten haben, können projectivisch so auf einander bezogen 
werden, dass je zwei Büschel, deren Mittelpunkte mit dem Punkte $P$ oder mit dem Punke II and also auch mit dem Vittelpunkte eines dritten Büschels in einer und derselben Geraden liegen, ein gerades Gebilde erzeugen, welches im ersten F'alle in derjenigen Geraden $\pi$, im letzteren aber in derjenigen Geraden $p$ liegt, welche vom Mittelpunkte des dritten Büschels harmonisch getrennt ist. Je drei Büschel, deren Mittelpunkte in einer und derselben von den sechs Curven $k_{1}, k_{2}, \ldots, k_{6}$ liegen, erzeugen diese Curve. Es sind hierdurch auch die sechs erwähnten Curven auf die Büschel und daher auch auf einander projectivisch bezogen, und zwar auf die im Satze angegebene Weise. Die Curve $k$ geht immer durch die vier Punkte $x y, x_{1} y_{1}, x y_{1}, x_{1} y .^{.6}$

„12. Wenn zwei Curven zweiter Ordnung zwei einander gegenüber liegende Eckpunkte eines vollständigen Vierseits mit einander gemein haben und jede auch noch durch zwei außerhalb der anderen liegende Eckpunkte des Vierseits geht, so schneiden sich die Geraden, welche die eine, gleichviel welche, von den beiden Curven in jenen gemeinschaftlichen Punkten beriuhren, and die Geraden, welche den anderen in den übrigen gemeinschaftlichen Punkten sich anschmiegen, in einem und demselben Punkte. Eine solche Lage hat, was die in der vorigen Nummer betrachteten Curven anbelangt, jede der drei Curven $k_{1}, k_{3}, k_{5}$ zu jeder der drei Curven $k_{2}, k_{4}, k_{6}$, und jede dieser sechs Curven zu jeder Curve $k$ zweiter Ordnung, welche durch sechs homologe Punkte 1, 3, 5, $2,4,6$ derselben geht.

Es schneiden sich daher die drei Geraden, welche die Curven $k_{1}, k_{3}, k_{5}$ in den Punkten $1,3,5$ berühren, in dem Punkte $T_{135}$, welcher in Hinsicht auf die Curve $k$ der. Pol der Geraden $t_{155}$ und zugleich der Pol des Dreieckes 246 ist. Die drei Geraden aber, welche die Curven $k_{2}, k_{4}, k_{6}$ in den Punkten 2, 4, 6 berïhren, schneiden sich in dem Punkte $T_{246}$, welcher vom Punkte $T_{135}$, durch die Punkte $P$, II harmonisch getrennt und in Hinsicht auf die Curve $k$ der Pol der Geraden $t_{246}$ des Dreieckes 135 ist.

Bewegen sich die sechs Punkte $1,2, \ldots, 6$ in den sechs Curven $k_{1}, k_{2}, \ldots, k_{6}$ als homologe Punkte derselben, so drehen sich die neun Geraden, welche die Eckpunkte des Dreieckes 135 mit den Eckpunkten des Dreieckes 246 verbinden, um die neun Punkte $p^{\prime} \pi^{\prime}$, während die Geraden $35,51,13$ um die Punkte $K_{1}^{\prime}, K_{3}^{\prime}, K_{5}^{\prime}$ und die Geraden 46,62, 24 und die Punkte $K_{2}^{\prime}, K_{4}^{\prime}, K_{6}^{\prime}$ sich drehen.

Die Geraden $T_{246} 1, T_{246} 3, T_{246} 5, T_{135} 2, T_{135} 4, T_{135} 6$, welche die Curve $k$ in noch sechs homologen Punkten der Curven $k_{1}, k_{3}, k_{5}$, $k_{2}, k_{4}, k_{6}$ schneiden, drehen sich um die Punkte, welche in Hinsicht auf diese Curven die Pole der Geraden PII sind. Fällt irgend einer der drei Punkte $1,3,5$ in die Gerade $t_{\mathbf{2 4 6}}$, so fallen alle drei in diese Gerade, die drei Punkte 246 aber in die Gerade $t_{135}$, so dass alsdam die Curve $k$ in den Inbegriff von zwei Geraden übergeht. 
Die Aufgabe, in eine gegebene Curve zweiter Ordnung, welche (11) durch die vier Punkte $x y, x_{1} y_{1}, x y_{1}, x_{1} y$ geht, zwei Dreiecke $135,246 \mathrm{zu}$ beschreiben, so dass die Pascal'schen Geraden der Sechsecke 163254, 123456, a. s. w. mit den gegebenne Geraden $p^{\mathrm{I}}, p^{\mathrm{II}}$, u. s. w. zusammenfallen, lässt nach dem Obigen zwei Auflösungen zu."

8. Nach diesen schönen Sätzen der Geometrie wollen wir zeigen, wie man das Steiner'sche Gegenpunktepaar $P$, $\Pi$ und die durch dieselben gehenden Pascal'sche Geraden $p^{\mathrm{I}} p^{\mathrm{II}} p^{\mathrm{III}}, \pi^{\mathrm{IV}} \pi^{\mathrm{V}} \pi^{\mathrm{VI}}$ des Sechseckes 123456 construieren kann, obne die Schnittpunkte der Gegenseiten desselben zu benützen.

Die Tangenten in den Eckpunkten eines einem Kegelschnitt einbeschriebenen Dreieckes, schneiden die Gegenseiten desselben in drei Punkten einer Geraden. Diese Grade, sowie ihren Pol nennt staudt "die Polare und den Pol des Dreieckes in Hinsicht auf den Kegelschnitt. Es sind also die Geraden $t_{246}, t_{135}$ und die Punkte $T_{246}, T_{135}$ der früheren Figur die Polaren und Pole der Dreiecke 135 , beziehungsweise 246 in Hinsicht auf den beiden Dreiecken umschriebenen Kegelschnitt $k$.

Nach Früherem sind diejenigen conjugierten Pole in Hinsicht auf $k$, welche die Punkte $T_{135}, T_{246}$ harmonisch trennen, die Steiner'schen Gegenpunkt $P$, II. Dieselben werden .von $k$ stets getrennt, da der Pol der Geraden $\left|T_{135} T_{246}\right|$, als Schnittpunkt der stets außerhalb $k$ liegenden Geraden $t_{135}, t_{246}$, außerhalb $k$ liegt.

Die neun Geraden $K_{i}^{\prime} K_{b}^{\prime}$, welche die Punkte $K_{1}^{\prime} K_{3}^{\prime} K_{5}^{\prime}$ mit den Punkten $K_{2}^{\prime} K_{4}^{\prime} K_{6}^{\prime}$ der früheren Figur, verbinden, werden entsprechend von den neun Geraden $i l$, welche die Eckpunkte des Dreieckes 135 mit den Eckpunkten des Dreieckes 246 verbinden, in den neun Punkten $\left(p^{\prime} \pi^{\prime}\right)$ geschnitten. Und die neun Punkte, welche auf den neun Geraden $\left|K_{i}^{\prime} K_{l}^{\prime}\right|$ die Punkte $\left(p^{\prime} \pi^{\prime}\right)$ von den Punkten $K_{i}^{\prime}, K_{l}^{\prime}$ harmonisch trennen, sind die neun Punkte $(p \pi)$; von letzteren Punkten liegen sechsmal drei in den Pascal'schen Geraden $p^{\mathrm{I}} p^{\mathrm{II}} p^{\mathrm{III}} \pi^{\mathrm{IV}} \pi^{\mathrm{V}} \pi^{\mathrm{VI}}$. Man kann also die Punkte $P$, II mittelst einer quadratischen Construction unabhängig von den Geraden $p^{\mathrm{T}} p^{\mathrm{II}} \ldots \pi^{\mathrm{VI}}$ und die Geraden selbst mittelst linearen Constructionen, ohne die Schnittpunkte der Gegenseiten des Pascal'schen Sechseckes aufzusuchen, erhalten.

Bezeichnet man die Tangenten des Kegelschnittes $k$ in den Punkten $12 \ldots 6$ mit $t_{1} t_{2} \ldots t_{6}$, so wird z. B. die Gerade 12, die Verbindungsgerade $K_{1}^{\prime} K_{2}^{\prime}$ der Punkte $K_{1}^{\prime}=\left(t_{1}, 35\right), K_{2}^{\prime}=\left(t_{2}, 26\right)$ in dem Punkte $\left(p^{\mathrm{I}} \pi^{\mathrm{VI}}\right)^{\prime}$ treffen, und der von $\left(p^{\mathrm{I}} \pi^{\mathrm{VI}}\right)^{\prime}$ durch $K_{1}^{\prime} K_{2}^{\prime}$ harmonisch getrennte Punkt $\left(p^{\mathrm{I}} \pi^{\mathrm{VI}}\right)$ gehört den Pascal'schen Geraden $p^{\mathrm{I}}, \pi^{\mathrm{VI}}$ an.

Die von den Punkten $K_{1}^{\prime}, K_{2}^{\prime}$ an $k$ gezogenen Tangenten berühren $k$ in den Punkten $11^{\prime}, 22^{\prime}$. Der Schnittpunkt $\left(11^{\prime}, 22^{\prime}\right)$ der 
Berührungssehnen ist der Pol der Geraden $K_{1}^{\prime} K_{2}^{\prime}$, und da sich die Geraden $12, K_{1}^{\prime} K_{2}^{\prime}$ in dem Punkte $\left(p^{\mathrm{I}} \pi^{\mathrm{VI}}\right)$ treffen und $\left(p^{\mathrm{I}} \pi^{\mathrm{VI}}\right)^{\prime}$ $\left.p^{\Upsilon} \pi^{\mathrm{Vl}}\right)^{\prime}$ conjugierte Pole sind in Hinsicht auf $k$, so geht die Gerade $1^{\prime} 2^{\prime}$ ebenfalls durch $\left(p^{\mathrm{T}} \pi^{\nabla I}\right)$, während sich die Geraden $1^{\prime} 2$, $12^{\prime}$ im Punkte $\left(p^{\mathrm{T}} \pi^{\mathrm{VI}}\right)$ schneiden.

Daraus können wir schließen, da dies bei allen neun Verbindungsgeraden der Punkte $K_{1} K_{3}^{\prime} K_{5}^{\prime}$ mit den Punkten $K_{2}^{\prime} K_{4}^{\prime} K_{b}^{\prime}$ stattindet, dass $p^{\mathrm{\gamma}} p^{\mathrm{Tl}} p^{\mathrm{III}} \pi^{\mathrm{IV}} \pi^{\mathrm{V}} \pi^{\mathrm{VI}}$ anch Pascal'sche Geraden des Sechseckes $1^{\prime} 2^{\prime} 3^{\prime} 4^{\prime} 5^{\prime} 6^{\prime}$, und $\left(p^{\mathrm{I}} p^{\mathrm{II}} p^{\mathrm{III}} \pi^{\mathrm{IV}} \pi^{\mathrm{V}} \pi^{\mathrm{VI}}\right)^{\prime}$ Pascal'sehe Geraden der Sechsecke 12'34'56', 1'2 3'45'6, sind. Daher:

Sind 135, 246 zwei beliebige, einem Kegelschnitt $k$ einbeschriebene Dreieeke und hat das Dreieck $1^{\prime} 3^{\prime} 5^{\prime}$ mit dem ersten, das Dreieck $2^{\prime} 4^{\prime} 6^{\prime}$ mit demzweiten Dreieck in Hinsicht auf $k$ je einen gemeinsamen Pol, und werden diese Pole von den conjugierten Polen $P, \Pi$ in Hinsicht a f $k$ harmonisch getrennt, so ist $P$,II ein Steiner'sches Gegenpunktepaar für die vier Paseal'seben Sechsecke 123456, 1'2'3'4'5'6',12'34'56', 1'2345'6. Die sechs durch $P$, II gehenden Pascal'schen Geraden $p^{\mathrm{I}} p^{\mathrm{II}} p^{\mathrm{II}}, \pi^{\mathrm{VV}} \pi^{\mathrm{V}} \pi^{\mathrm{VI}}$ des ersten Sechseckes fallen mit sechs Pascal'schon Geraden des zweiten Sechseckeszusammen, und ebensofallendie sechs durch $P$, II gehenden Pascal'schenGeraden $p^{\prime I} p^{\prime I I} p^{\prime I I I} \pi^{\prime I V} \pi^{\prime \nabla} \pi^{\prime \nabla I}$ desdritten Sechseckes mit sechs Pascal'scbenGeraden des vierten Sechseckes zusammen. Je zwei der drei ersten Geraden $p o d e r \pi$ werden vonderdrittendurcheineder Geraden $p^{\prime}$ beziehungsweise $\pi^{\prime}$ harmonisch getrennt.

9. Da, wie wir sahen, die Verbindungsgerade eines Steiner'schen Gegenprunktepaares den Kegelschnitt immer in reellen Punkten schneidet, so liegen von den zwanzig Steiner'schen Punkten zehn innerhalb und ihre Gegenpunkte a a Berhalb des dem Pascal'schen Sechseck umschriebénen Kegelschnittes.

Man kann aber auch im vorhinein bestimmen, welcher der Steiner'schen Pankte eines Gegegenpunktepaares innerhalb und welcher außerhalb des Kegelschnittes $k$ liegt.

Hält man bei einem Pascal'schen Sechsecke drei nicht aufeinander folgende Eckpunkte fest, so ergeben die drei ibnen gegenüber liegenden Eckpunkte secbs Permutationen, von welchen drei und die übrigen drei cylklische Permutationen sind. Die Pascalschen Geraden der ersten drei, und der anderen drei Sechsecke schneiden sich in zwei Steiner'schen Punkten, welche Gegenpunkte sind. So z. B. schneiden sich die Pascal'schen Geraden der Sechsecke

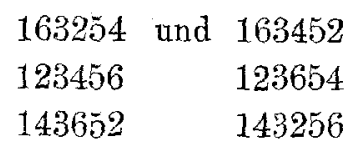

in den Steiner'schen Gegenpunkten $P, \Lambda$. 
Es ist nun zu unterscheiden, welcher der Steiner'schen Punkte aufaerbalb des Kegelschnittes $k$ liegt, denn der andere liegt dann innerhalb desselben.

Ich behaupte: Wenn drei nicht auf einander folgende Eckpunkte. und dieihnengegenüber liegenden Eckpunkte eines Pascal'schen Sechseckes auf den durchdieselbengehenden Kegelschnittindemselben Sinne auf einander folgen, so geht die, diesem Pascal'schen Sechsecke zugehörige Paseal'sche Ge. rade durch einen außerhalb des Megelschnithes lie. genden Steiner'schen Punkt.

Es möge beim Pascal'schen Sechsecke 123456 der Sinn der Eckpunkte 135, 246 auf den umschriebenen Kege'schnitt $k$ derselbe sein, dann können die drei letzteren Punkte in Bezug auf die drei ersteren nur folgende drei Lager haben: 1. Von den Punkten 246 ist keiner von den beiden anderen durch zwei der Punkte 135 getrennt; 2. ein Punkt von 246 wird ron den beiden anderen durch einen Punkt und die beiden anderen von 135 getrennt; endlich 3. je zwei der Punkte 246 werden durch zwei der Punlkte 135 getrennt. Im 1, 2, 3. dieser Fälle haben beziehungsweise ein, zwei, drei Pascal'sche Geraden der Sechsecke mit dem umschriebenen Kegelschnitt $k$ keinen reellen Punkt gemein, also gehen in jedem Falle die Pascal'schen Geraden dieser Sechsecke durch einen auferhalb des Kegelscbnittes $k$ liegenden Steiner'schen Punkt.

10. Sechs Punkte 123456 eines Kegelschnittes $k$ bilden die Eckpunkte von zwanzig Dreiecken oder zehn Paar Ergänzungsdreiecken, wenn je zwei jener Dreiecke, die keirien gemeinsamen Eckpunkt haben, so nennt. Die Pole und Polaren jedes der Dreiecke in Hinsicht auf $k$, wollen wir wie fruber mit $T_{i j l^{2}}$, und $t_{i j t}$ bezeichnen, wo die Zeiger ijl mit den Eekpunkten des Ergänzungsdreieckes übereinstimmen.

Von den zwanzig Dreiecken haben fünfzehnmal vier, zwei gemeinsame Eckpunkte; z. B. haben die Dreiecke 123, 124, 125 , 126 die gemeinsamen Eckpunkte 12. Untersuchen wir die Lage ihrer Pole $T_{456}, T_{346}, T_{345}$.

Die sechs Tangenten $t_{1} t_{2} t_{3} t_{4} t_{5} t_{6}$ ron $k$ in den Punkten 123456 schneiden die zwei Tangenten $t_{1}, t_{2}$, in den sechs Punkten von zwei projectiven Punktreihen, und die Verbindungsgeraden dieser. Schnittpunkte mit den Punkten 2, beziehungsweise 1, geben projective Strahlen; also ist

$$
2\left(1\left(t_{1} t_{2}\right)\left(t_{1} t_{3}\right)\left(t_{1} t_{4}\right)\left(t_{1} t_{5}\right)\left(t_{1} t_{6}\right)\right) \pi 1\left(\left(t_{2} t_{1}\right) 2\left(t_{2} t_{3}\right)\left(t_{2} t_{4}\right)\left(t_{2} t_{5}\right)\left(t_{2} t_{6}\right)\right) .
$$

Es liegen daher die Schnittpunkte

$$
12 T_{456}^{\prime} T_{356} T_{346}^{r} T_{34 \check{s}}
$$

der sechs entprechenden Strahlenpaare auf einen Kegelschnitt $k_{12}$, der $k$ in den Puakte 12 berührt, und für die auf $k$ und $k_{12}$ liegenden sechs Punkten ist

$$
123450 \pi 12 T_{456} T_{356} T_{346} T_{345}
$$


Die zwei Kegelschnitte $k, k_{12}$ liegen in Bezug auf die gemeinsame Berührungssehne 12 und den Schnittpunkt $\left(t_{1} t_{2}\right)$ der gemeinsamen Tangenten $t_{1}, t_{2}$, als Collineations-Axen und Mittepunkt perspectiv; dabei entsprechen obige sechs Punkte einander. Die sechs Seiten des Viereckes $T_{12}^{3}=T_{456} T_{356} T_{346} T_{345}$ gehen durch die Schnittpunkte der sechs Seiten des Viereckes 3456 mit der Geraden 12, d. h. durch sechs von den fünfundvierzig Pascal'schen Punkten, wenn man diejenigen Punkte Pascal'sche Punkte nennt, in welchen sich die fünfzehn Seiten eines vollständigen Pascal'schen Sechseckes, außer ihren Eckpunkten, schneiden. Durch jeden dieser sechs Pascal'schen Punkte auf der Berührungssehne 12, geht aber noch je eine Seite der (dem obigen Viereck $T_{12}$ entsprechend entstandenen) Vierecke $T_{34}, T_{56}, T_{35}, T_{46}$, $T_{36}, T_{45}$, welche denjenigen Kegelschnitten $k_{34}, k_{56}, \ldots, k_{45}$ einbeschrieben sind, die $k$ in je zwei Eckpunkten des Viereckes 3456 berühren Daher:

Sechs Punkte 123456 eines Kegelschnittes $k$ bilden die Eckpunkte von zwanzig Dreiecken, welche in Hinsicht a u $k z$ wanzig. Pole haben. Vondiesen Polen liegen fünfzehnmal vier auf solchen Kegelsehnitten $k_{i j}$, welchek in $z$ wei Punkten $i j$ der sechs Punkte berühren. Die vier Pole and die Berührungspunkte entsprechen projectiv den sechs angenommenen Punkten und $k_{i j}, k$ sind bei dieser projectiven Beziehung perspectiv in Bezug auf die gemeinsame Berührungssehne $i j$ und ibren Pol.

Die sechs Seiten des Viereckes $T_{i j}$, dessen Eckpankte die vier auf $k_{i j}$ liegende Pole sind, gehen durch die sechs auf der Berübrungssehne ij liegenden Pascal'schen Punkte. Es gibt fünfzehn solche Vierecke $T_{i j}$, entsprechend den fünfzehn Kegelschnitten $k_{i j}$; ihre neunzig Seiten $u$ gehen parrweise dureh die fünfundvierzig Pascal'sohen Punlte des Sechseckes 123456 ; durch jeden der zanzig Pole gehen neun solche Geraden $u$.

11. Betrachten wir jetzt zwei von den fünfzehn Kegelschnitten $k_{i j}$, welche $k$ in denselben von den sechs Punkten $12 \ldots 6$ berühren; z. B. die Kegelschnitte $k_{12}, k_{13}$. Diese berühren also $k$ in den Punkten 1, 2, beziehungsweise 1,3 , und gehen beide durch den Pol $T_{456}$. Sie haben in dem Punkte 1 eine dreipunktige Berührung; denn der vierte Schnittpunkt müsste den Scbnittpunkt $T_{456}$ von den Berührungssehnen 12,13 harmonisch trennen. Aber alle Punkte, welche $T_{456}$ ron den Geraden 12, 13 harmonisch tremen, liegen in der Tangente $t_{1}$, welche die drei Kegelschnitte $k, k_{12}, k_{13}$ in dem Punlst 1 berührt; also fällt jener vierte Schnittpunkt ebenfalls in diesen Punkt 1. 
Betrachtet man ferner die drei Kegelschnitte $k_{12}, k_{93}, k_{31}$, welche $k$ in je zwei der Eckpunkte des Dreieckes 123 berühren, so sieht man, dass sie alle durch den Pol $T_{456}$ des Dreieckes 123 in Hinsicht auf $k$ gehen und sich gegenseitig in den Eckpunkten dieses Dreieckes dreipunktig berühren.

Die fünfzehn Kegelschnitte $k_{i j}$ reihen sich in zwanzig solehen Tripeln von Kegelschnitten, entsprechend der Anzahl der Dreiecke, in welche man das Sechseck $12 \ldots 6$ zerlegen kann. Also:

Von den fünfzehn Kegelschnitten $k_{i j}$, welcheden Kegelsehnitt $k$ in jezwei der sechs Punkte 123456 berühren und a uberdem durch je vier der Poleder zwanzig Dreiecke $\delta$, deren Eckpunkte die Punkte $12 \ldots 6$ sind, gehen, berühren sich in jedem dieser Punkte fünf dreipunktig.

Je drei der Kegelschnitte $k_{i j}$, welche $k$ in den Eckpunkten eines Dreieckes $\delta$ berühren, haben in diesen Eckpunkten paarweise eine dreipunktige Berührung, und schneiden sich auberdem in dem Pole des Dreieckes in Hinsicht a uf $k$. Die neun Geraden $u$, welche den gemeinsamen Pol mit den ubrigen neuen auf diesen drèi Kegelschnitten $k_{i j}$ liegenden Polen verbinden, gehen durch diejenigen auf den Seiten des Dreiekes ó liegenden neun Pascal'schen Punkten, welche den Seiten des Ergänzungsdreieckes von $\delta$ nicht angehören. Satz:

Beiläufig folgt der möglicher Weise noch nicht bekannte

Sind $A B C$ die Eckpankte eines Dreieckes, $T$ ein beliebiger Punkt einer Ebene, und trennen die Geraden $t_{a} t_{b} t_{c}$ den Punkt $T$ von den Seitenparen des Dreieckes harmonisch, so werden sich die drei Kegelschnitte, wolehe durch $T$ gehen and je zwei Seiten des Dreiseits $t_{a} t_{b} t_{e}$ in den Eckpunkten des Dreieckes $A B C$ berühren, in diesen Eckpunkten, dreipunktig berühen. Der Punkt $T$ muss nicht innerhalb des Dreieckes $A B C$ liegen.

12. Betrachten wir jetzt zwei der Kegelschnitte $k_{i j}$, die keine gemeinsamen Berührungspunkte haben, z. B. $k_{12}, k_{34}$. Der erste berührt $k$ in den Punkten 1,2 und geht durch die Pole $T_{356}, T_{456}$, $T_{346}, T_{345}$; der zweite berührt $k$ in den Punkten 3,4 und geht noch durch die Pole $T_{156}, T_{256}, T_{125}, T_{126}$.

Die Vierecke 1234, $T_{1{ }^{6} 6} T_{256} T_{356} T_{45,}$, und das Vierseit $t_{1} t_{2} t_{3} t_{4}$, dessen Seiten $k$ in den Eckpunkten des ersten Viereckes berühren, haben ein gemeinsames Diagonaldreieck $z_{56} z_{56}^{\prime} z_{56}^{\prime \prime}$, da die Gegenseiten 


$$
\begin{aligned}
& T_{156} T_{256}, T_{256} T_{456} \text { durch den Pascal'schen Punkt }(12,34) \\
& T_{15.6} T_{356}, T_{956} T_{456} \quad \text { " } \quad \text { " } \\
& T_{156} T_{456}, T_{256} T_{355} \quad n \quad n \quad \text { " } \quad \text { " }
\end{aligned}
$$

gehen.

Daraus folgt, dass die zwei Kegelschnitte $k_{12}, k_{34}$ in Bezug auf die zwei Diagonalen

$$
\begin{aligned}
& z_{56}=|(13,24)(12,34)|=\mid\left(t_{1} t_{4}\right)\left(t_{2} t_{3}\right), \\
& \left.z_{56}^{\prime}=|(12,34)(14,23)|=\mid\left(t_{1} t_{3}\right)\left(t_{2} t_{4}\right)\right\}
\end{aligned}
$$

und die ihnen gegenüberliegenden Eckpunkte

$$
\left(z_{56}^{\prime} z_{5 j}^{\prime \prime}\right)=(14,23), \quad\left(z_{56}^{\prime \prime} z_{56}\right)=(13,24)
$$

des Diagonaldreieckes $z_{56} z_{56}^{\prime} z_{56}^{\prime \prime}$, als Involutionsaxe und Mittelpunkt centrisch-involutorisch liegen.

Denn es werden z. B. von der ersten Diagonale und dem ihr gegenüber liegenden Diagonalpunkt harmonisch getrennt, die Punktepaare 14, 23, $T_{156} T_{456}, T_{256} T_{356}$ und die Geradenpaare $t_{1} t_{4}, t_{2} t_{3}$, welche entsprechende Punkte und Geraden jener Kegelschnitte sind.

Diese Involutions-Mittelpunkte und Involutions-Axen sind die stets reellen Contingenzpunkte und gemeinsamen Sehnen der Kegelschnitte $k_{12}, k_{34}$; sie trennen die Berührungssehnen 12,34 der Kegelschnitte $k k_{12}, k_{34}$ harmonisch, und sind conjugierte Pole und Polaren in Hinsicht auf $k$. Also:

Je zwei der fünfzehn Kegelschnitte $k_{i j}$, welche $k$ in vier verschiedenen, also in zwei und zwei, der Eckpunkte des Sechseckes $12 \ldots 6$ berühren, sind centriseh-involutorisch in Bezug auf zwei Diagonalpunkte und Diagonalen, als Involutions-Mittelpunkte und Involutions-Axen desjenige Viereckes, dessen Eckpunkte jene vier Berührungsunkte sind. Diese Diagonalpunkte und Diagonalen, sind die stets reelen Contingenz. punkte und gemeinsame Sehnen der zwei Kegelschnitte $k_{i j}$; sie trennendie zwei Berührungssehnen der zwei Kegelschnitte harmonisch und sind conjugierte Pole und Polaren in Hinsicht a uf $k$.

Für die Kegelschnittpaare $k_{12} k_{34}, k_{23} k_{41}, k_{13} k_{24}$ welche $k$ in den Punktepaaren 12, 34; 23,41;13,24 berühren, sind die stets reellen gemeinsamen Sehnenpaare $z_{56} z_{56}^{\prime}, z_{56}^{\prime} z_{56}^{\prime \prime}, z_{56}^{\prime \prime} z_{56}$ je zwei Seiten des zum Viereck 1234 und Vierseit $t_{1} t_{2} t_{3} t_{4}$ gehörigen Diagonaldreieckes $z_{56} z_{56}^{\prime} z_{56}^{\prime \prime}$

Da das Sechseck 123456 in fünfzehn solche Vierecke 1234, welche mit dem Sechsecke vier gemeinsame Punkte haben, zerfällt, und die Seiten ihrer Diagonaldreiecke die stets reellen gemeinsamen 
Sehnen sind von zwei der fünfzehn Kegelschnitte $k_{i j}$, so haben diese fünfzehn Kegelschnitte fünfundvierzig solche reelle gemeinsame Sehnen. (Wir bemerken, dass wir die etwa vorhandenen anderen gemeinsamen Sehnen, auch wenn sie reell sind, hiebei nicht mitzählen.) Daher:

Die stets reellen gemeinsamen Sehnen der fünfzehn Kegelschnitte $k_{i j}$, sind die fünfundvierzig Diagonalendesjenigen Brianchon'schen Sechsseits $t_{1} t_{2} \ldots t_{6}$, dessen Seiten den Kegelschnitt $k$ in den sechs Eckpunkten des Pascal'schen Sechseckes 123456 berühren. A us jedem Pascal'schen Punktestrahlen zwei gemeinsame Sehnen aus and trennen zwei Seiten des Sechseckes harmonisch.

13. Drei Kegelschnitte $k_{i j}$, welche $k$ in sechs verschiedenen Punkten berühren, haben drei Paar stets reelle gemeinsame Sehnen.

Nehmen wir die Kegelschnitte $k_{12}, k_{34}, k_{56}$; von diesen haben $k_{34} k_{56}$ die gemeinsamen Sehnen $z_{12}=\left(t_{3} t_{6}\right)\left(t_{4} t_{5}\right), z_{12}^{\prime}=\left(t_{3} t_{5}\right)\left(t_{4} t_{6}\right)$, $k_{56} k_{12} \quad " \quad n \quad z_{34}=\left(t_{5} t_{2}\right)\left(t_{6} t_{1}\right), z_{34}^{\prime}=\left(t_{5} t_{1}\right)\left(t_{6} t_{2}\right)$, $k_{12} k_{31} \quad n \quad n \quad n \quad z_{56}=\left(t_{1} t_{4}\right)\left(t_{2} t_{3}\right), z_{56}^{\prime}=\left(t_{1} t_{3}\right)\left(t_{2} t_{4}\right)$,

und es schneiden sich

$$
\begin{array}{lccccc}
z_{12} z_{34} z_{56} & \text { als } & \text { Hauptdiagonalen } & \text { des Sechseits } & t_{1} t_{6} t_{3} t_{2} t_{5} t_{4} \\
z_{12} z_{34}^{\prime} z_{56}^{\prime} & n & n & n & " & t_{1} t_{3} t_{6} t_{2} t_{4} t_{5} \\
z_{12}^{\prime} z_{34} z_{56}^{\prime} & n & n & n & n & t_{1} t_{6} t_{4} t_{2} t_{5} t_{3} \\
z_{12}^{\prime} z_{34}^{\prime} z_{56} & n & n & n & n & t_{1} t_{4} t_{6} t_{2} t_{3} t_{5}
\end{array}
$$

in je einem Brianchon'schen Punkte. Jene gemeinsamen Sehnen bilden daher ein Vierseit $z^{\mathrm{II}, \mathrm{III}}$, dessen Diagonaldreieck $\triangle^{\mathrm{II} \text { III }}=$

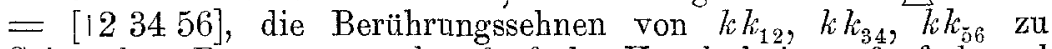
Seiten hat. Da man von den fünfzehn Kegelschnitten fünfzehnmal drei solche auswählen kann, welche $k$ in allen sechs Punkten $12 \ldots 6$ berühren, so folgt:

Die fünfzehn Kegelschnite $k_{i j}$, theilen sich in fünfzehn solche Tripeln von Kegelschnitten, welche den Kegelschnitt $k$ in allen sechs Eckpunkten des Sechseckes 123456 ber ühren. Ihre füntund vierzig stets reellen gemeinsamen Sehnen sind die Seiten von fünfzehn Vierecken. Diese Vierecke sind Polvierecke (d. h. die Gegenseiten sind conjugierte Polaren) in Hinsicht auf $k$; ihre sechzig Eckpunkte sind die sechzig Brianchon'sche Punkte desjenigen Sechsseits, dessen Seiten $k$ in den Punkten $12 \ldots 6$ berühren, endlich sind ihre Diagonaldreiecke diejenigen fünf- 
zehn Dreiecke, a uf dessen Seiten alle sechs Punkte $12 \ldots 6$ liegen.

Ferner: Die 45 Diagonalen eines vollständigen Brianchon'schen Sechsseits $t_{1} t_{2} t_{3} t_{4} t_{5} t_{6}$, dessen Seiten elnen Kegelschnitt $k$ in den sechs Punkten eines Pascal'schen Sechseckes 123456 berühren, spalten sich in Hinsicht auf $k$ in 15 Polvierecke, deren Eckpunkte die 60) Brianchon'schen Punkte sind, und in 15 Poldreiecke. Ebensobilden die 45 Pascal'schen Pankte die Eckpunkte $\nabla \circ n$ 15 Polvierseiten, deren Seiten die 60 Pascal's che Geraden sind, und in 15 Poldreiseiten. Die Diagonaldreiecke jener 15 Polvierecke haben bIoß 15 Seiten, welche mit den 15 Seiten des Pascal'schen Sechseckes zusammenfallen; ebenso sind die 15 Eckpunkte des Brianchon'schen Sechsseits die 15 Eckpunkte der Diagonaldreiecke dieser 15 Polvierseiten. 